\title{
EDITORIAL
}

\section{Focus on RNA analysis}

\author{
Sapna Deo
}

Published online: 19 April 2009

(C) Springer-Verlag 2009

Until the 1990s, RNA was regarded only as a messenger between DNA and protein. The double helix of DNA was mostly getting the publicity on covers of magazines and other media, becoming a symbol of life. It was the discovery that RNA can act as a catalyst, which changed the perception of the role of RNA, that earned the Noble Prize for Professor Sidney Altman at Yale University and Professor Thomas Cech at the University of Colorado in 1989. The concept of a ribozyme, RNA that is able to catalyze its own replication and the synthesis of other RNA molecules, was proposed by these scientists. This led to an idea that RNA may be the first genetic material on earth, creating the concept of the "RNA world". The property of RNA to act as both genes and enzymes could offer a solution to the "chicken-and-egg" problem, supporting the belief that the "RNA world" could be the original pathway to cells. This also suggested that RNA could play a more active role in gene expression than previously thought. A flurry of research activity in the field of RNA followed this discovery, and earned for RNA a pivotal position in cellular biology. Another breakthrough in RNA research was the discovery that small double-stranded RNA (dsRNA) could trigger silencing of a complementary messenger RNA sequence in the nematode Caenorhabditis elegans, a process termed "RNA interference" (RNAi). By use of artificial dsRNA, the RNAi mechanism was also shown to work in mammalian cells, opening up a new era in basic

S. Deo $(\bowtie)$

Department of Chemistry and Chemical Biology,

Indiana University Purdue University Indianapolis,

LD326 Blackford Street,

Indianapolis, IN 46202, USA

e-mail: sdeo@iupui.edu research and disease therapy. This work was honored in 2006 when Professor Andrew Z. Fire from Stanford University and Professor Craig C. Mello from the University of Massachusetts Medical School in Worcester received the Noble Prize for Medicine. The discovery of RNAi has added further complications to our understanding of gene regulation and, consequently, has created new opportunities in this research field. In the same year, the Noble Prize for Chemistry was awarded to Professor Roger D. Kornberg for determining how RNA molecules convert genetic information stored in DNA into working proteins. These two Noble awards in the same year have attracted much deserved attention to the field of RNA.

RNA molecules are now viewed in two different ways - as protein-coding RNAs and as noncoding RNAs. The small non-coding RNAs ( $<35$ nucleotides in length) are classified into categories such as microRNAs (miRNAs), PIWIassociated RNAs, and small interfering RNAs (siRNAs), on the basis of their function, length, biogenesis, structural and/or sequence features, and protein-binding partners. Much scientific interest is now focused on the small noncoding RNAs, because they have been found to play a major role in gene expression. It is now well accepted that double-stranded small RNAs synthesized within the cell can alter and inhibit gene activity by RNAi-like mechanisms; this ultimately controls the development and physiological functions of cells and organisms. This discovery has had a tremendous impact on biomedical research and will ultimately lead to new therapies in medicine. In 2000 it was discovered that small nucleotidelength RNAs called microRNAs (16-25 nucleotides in length) previously regarded as the dead matter of cells are regulators of gene expression. Deregulation in microRNA expression is now linked to a variety of diseases, most notably cancer. RNA, once regarded as merely a messenger molecule between DNA and protein, has now emerged as a promising target for 
cancer therapy and diagnosis. The RNA molecule, whether in the form of messenger RNA or microRNA, performs a variety of essential functions and is the target of study in fundamental biomedical research, drug discovery, and diagnostics.

Advances in any emerging areas of research need fueling from many different disciplines of sciences including analytical chemistry. For example, advances in genomic and proteomics studies were possible because of developments in analytical technologies. To tackle any environmental or man-made disasters safely and efficiently we need reliable analytical methods. On a similar note, advances in the field of RNA have been made through the development of a variety of analytical methods. Methods such as microarrays, polymerase chain reaction, molecular beacons, hybridization, etc., have been developed that enable sensitive measurement with the capability of in-situ RNA detection. The information provided by RNA analysis is critical for understanding gene expression, biomarker identification, and response to therapy. However, there are many areas of critical need in RNA analysis that require our attention. These critical needs include the following:

- $\quad$ sensitive, high-speed, reliable, and simple class-specific RNA detection methods;

- the ability to visualize in real-time the level of expression and location of specific RNAs in living cells;

- methods for in-situ RNA analyses that can be adapted to larger scales; this will also include the development and use of multiple RNA detection probes;

- detection and quantification of rare and trace levels of RNAs;

- efficient RNA preparation to overcome RNA degradation and limited sample availability, and ability to completely remove genomic DNA contamination from the RNA;

- targeted RNA isolation, for example, isolation of microRNA from the total RNA preparation - in general, isolation of small RNAs from total RNA is a big challenge;

- methods for enhancing the stability of RNA and its efficient preservation in blood and tissue samples followed by RNA isolation;
- development of microfluidics technologies to expedite discovery and reduce the cost of analysis;

- methods to study RNA-small molecule and RNA-drug interactions;

- methods to study the structural complexity of RNA molecules;

- delivery or transfection of small RNAs to target cells, obtaining optimum stability of small RNA, studying toxicity levels of small RNA, and standardization of small RNA technologies.

Diverse approaches and methods are necessary for understanding of the role of RNA. Analysts working in teams with biomedical scientists, pharmacists, and engineers in interdisciplinary fields will enhance the pace of discovery in this field. The coming years are crucial for making great advances in our understanding of RNA function. These advances are possible only if matched by equal pace in the development of analytical technologies. In this issue of "Analytical and Bioanalytical Chemistry" you can read articles on RNA analysis. These articles focus on trends in small RNA analysis and applications of mass spectrometry and microarray technologies in RNA detection.

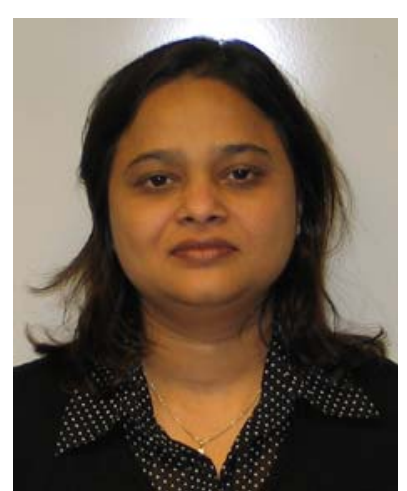

Sapna Deo has been an Assistant Professor of Bioanalytical Chemistry in the Department of Chemistry and Chemical Biology at Indiana University-Purdue University, Indianapolis, since 2005. She is author and coauthor of over 55 scientific publications and several patents and an editor of the book "Photoproteins in Bioanalysis". Dr Deo's research interest is in the development of novel bioanalytical techniques for detection of microRNAs and RNA molecules, based on luminescence detection, for application in diagnosis and pathogen detection. Other areas of research interest in Dr Deo's laboratory include development of molecular probes for biosensing and bioimaging applications. Dr Deo's research is funded by the National Science Foundation and the National Institutes of Health. 